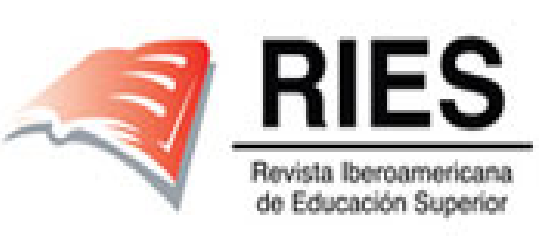

Revista Iberoamericana de Educación Superior

E-ISSN: 2007-2872

emmaro@unam.mx

Instituto de Investigaciones sobre la

Universidad y la Educación

México

Marúm-Espinosa, Elia; Reynoso-Cantú, Elsa-Laura

La importancia de la educación no formal para el desarrollo humano sustentable en México

Revista Iberoamericana de Educación Superior, vol. V, núm. 12, 2014, pp. 137-155

Instituto de Investigaciones sobre la Universidad y la Educación

.jpg, México

Disponible en: http://www.redalyc.org/articulo.oa?id=299129977008

- Cómo citar el artículo

- Número completo

- Más información del artículo

Página de la revista en redalyc.org

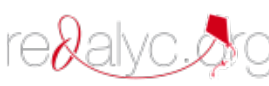

Sistema de Información Científica

Red de Revistas Científicas de América Latina, el Caribe, España y Portugal

Proyecto académico sin fines de lucro, desarrollado bajo la iniciativa de acceso abierto 


\title{
La importancia de la educación no formal para el desarrollo humano sustentable en México
}

\author{
Elia Marúm-Espinosa y Elsa-Laura Reynoso-Cantú
}

\section{RESUMEN}

Este artículo plantea la educación para adultos como un medio trascendental para expandir en la educación el desarrollo humano sustentable de nuestro país, por lo cual ésta ya no debe considerarse como una práctica compensatoria de deficiencias del sistema educativo escolarizado. Argumenta que la vulnerabilidad social que genera el rezago educativo actúa en al menos dos sentidos: por una parte, impide a la población con esta característica insertarse con mejores condiciones en la dinámica socioeconómica presente y futura y, por otra, sitúa en un entorno familiar con escaso capital socioeducativo a una buena parte de la población en edad escolar. Señala que el desarrollo humano sustentable no podrá alcanzarse sin la puesta en marcha de una decidida política educativa encaminada a lograr la máxima cobertura en la educación básica de los adultos que permita mejorar la calidad de la educación obligatoria de sus hijos, y permita mayor acceso a la educación media y superior a grupos más amplios de la población, que a la vez les dé acceso a actividades productivas de alto valor agregado sustentadas en el conocimiento, y conlleve a una ciudadanía responsable y participativa.

Palabras clave: educación para adultos, rezago educativo en México, desarrollo humano sustentable, educación básica y rezago educativo.

Elia Marúm-Espinosa

Mexicana. Doctora en Economía por la Universidad Nacional Autónoma de México (UNAM). Profesora investigadora y directora del Centro para la Calidad e Innovación de la Educación Superior del Centro Universitario de Ciencias Económico Administrativas (CUCEA), Universidad de Guadalajara, México. Temas de investigación: gestión de la educación superior, planeación de la educación, calidad y acreditación de la educación superior, género, liderazgo y equidad.

Elsa-Laura Reynoso-Cantú

Mexicana. Doctora en Ciencias Sociales con Orientación en Desarrollo Sustentable por el Instituto de Investigaciones Sociales de la Universidad Autónoma de Nuevo León (UANL), México. Colaboradora del Programa de educación ambiental de la Secretaría de Desarrollo Sustentable, UANL. Temas de investigación: medio ambiente y sustentabilidad, educación ambiental, análisis del discurso político, evaluación de políticas públicas e impacto medio ambiental. 


\section{A necessidade de educação não formal para o desenvolvimento humano sustentável no México}

\section{RESUMO}

O presente trabalho coloca a educação para adultos como meio transcendental para expandir a educação o desenvolvimento humano sustentável do país. Por esse motivo, a educação não deve ser mais considerada como prática compensatória de deficiências do sistema educativo escolarizado. Argumenta que a vulnerabilidade social gera o atraso educativo atual em pelo menos dois sentidos: de um lado, impede à população com esta característica de se inserir em melhores condições na dinâmica socioeconómica presente e futura e, do outro lado, coloca num ambiente familiar com escasso capital socioeducativo a uma grande parte da população em idade escolar. Salienta que o desenvolvimento humano sustentável não poderá ser atingido sem implementação de uma decidida política educativa destinada a conseguir máxima cobertura na educação básica dos adultos, que permita melhorar a qualidade da educação obrigatória dos filhos deles, e que possibilite maior acesso à educação média e superior para grupos mais amplos da população, para eles terem acesso a atividades produtivas de alto valor agregado, sustentadas no conhecimento, e a uma cidadania responsável e participativa.

Palavras chave: educação para adultos, atraso educativo no México, desenvolvimento humano sustentável, educação básica eatraso educativo.

\section{The need to non-formal education for the sustainable and human development of Mexico}

\section{ABSTRACT}

This article presents adult education as a significant means for expanding educational development for sustainable human development of our country. Therefore, this should be considered a compensatory practice for deficiencies of the scholastic educational system. We argue that the social vulnerability generated by educational lag works in at least two ways: in the first place, it prevents the population with this trait from placing themselves in better conditions within current and future socioeconomic dynamics; secondly, it places a large part of the school-age population in family milieus with limited socio-educational capital. The article points out that sustainable human development cannot be attained without setting in motion a decisive educational policy geared towards achieving maximum coverage in basic adult education, allowing them to improve the quality of compulsory education for their children; providing greater access to middle and higher education for broader groups in the population, in turn letting them access productive activities of high added value, supported by knowledge; and having a responsible and participative citizenry.

Key words: adult education, educational lag in Mexico, sustainable human development, basic education and educational lag.

Recepción: 23/02/13. Aprobación: 31/07/13. 


\section{Introducción}

La política educativa mexicana no da evidencias claras de estar armonizada con las exigencias del desarrollo humano sustentable de una de las economías más abiertas a la competencia internacional, y con un enorme potencial, como se ha planteado en el discurso oficial y en el Plan Nacional de Desarrollo y sus programas sectoriales y especiales relacionados con la educación, la ciencia y la tecnología (Presidencia de la República, 2007), pues se ha brindado una deficiente atención y se observa una fuerte reducción del presupuesto dedicado a la educación de adultos, espacio estratégico para la inserción exitosa en la vida social y productiva de un importante segmento de la población de México.

Las evaluaciones e información de los factores del entorno que se realizan en el marco de pruebas estandarizadas como las del Programa para la Evaluación Internacional de Alumnos (PISA, por sus siglas en inglés) que realiza la Organización para la Cooperación y el Desarrollo Económicos (OCDE), los Exámenes de Calidad y el Logro Educativo (EXCALE) que se aplican en los niveles preescolar, primaria y secundaria de planteles públicos y privados del país, que realiza el Instituto Nacional para la Evaluación de la Educación (INEE), demuestran que los rendimientos académicos más deficientes y la deserción escolar más alta en los diferentes niveles educativos, se asocian con niñas y niños provenientes de hogares encabezados por padres con baja escolaridad, por lo que la educación de adultos tiene una importancia más allá del individuo que la recibe, y es mayor su efecto multiplicador en el desarrollo nacional. Estos resultados se confirman con los obtenidos en la Evaluación Nacional de Logro Académico en Centros Escolares (ENLACE).

En la información oficial analizada tampoco encontramos evidencia que permita percibir que se esté haciendo un esfuerzo considerable frente al lastre que significa el gran rezago educativo de la población adulta para el desarrollo humano sustentable, pues la sitúa en condiciones de gran vulnerabilidad social, ya que la educación no formal o educación para adultos, tiene como finalidad última atender el rezago educativo y apoyar a quienes ya no están en condiciones o posibilidades de asistir a la escuela para obtener la formación y la capacitación necesarias, que les permitan el desarrollo adecuado de sus talentos en la vida productiva y social, pues a esta educación se le sigue considerando como un complemento (a veces poco relevante) de la educación formal.

Por ello, no sólo se le destinan proporcionalmente recursos presupuestales muy por debajo de cualquier otra modalidad educativa, sino que en años recientes estos recursos se han reducido de manera importante en términos nominales y reales, limitando así las posibilidades de avanzar en el combate al desempleo y la pobreza, y en el aporte educativo al desarrollo humano sustentable, ${ }^{1}$ lo que no es consistente y deja de lado la meta establecida hace más de tres lustros en el artículo tercero constitucional, de universalizar la educación básica en nuestro país, y nos aleja de cumplir con los compromisos internacionales adquiridos en el seno de la Organización de la Naciones Unidas (ONU, 2000), a través de los Objetivos del Milenio, de erradicar la pobreza extrema y el hambre, y lograr la enseñanza primaria universal.

Todo ello limita el desarrollo humano sustentable de México, entendido como el proceso permanente de ampliación de capacidades y libertades que permita a todos los mexicanos tener una vida digna sin comprometer el patrimonio de las generaciones futuras, y obstaculiza el avance de la construcción de las bases de la sustentabilidad económica, social y ambiental del desarrollo, resumidas en la reforma del propio artículo tercero, al señalar que la educación

\footnotetext{
${ }^{1}$ La importancia de la educación en el desarrollo humano sustentable consiste en ser un proceso a través del cual es posible iniciar, propagar, mantener y articular cada una de sus dimensiones. Para abundar en este planteamiento véase Elsa Reynoso-Cantú y Elia Marúm-Espinosa, 2010.
} 
que imparta el Estado será democrática, definiéndola "no solamente como una estructura jurídica y un régimen político, sino como un sistema de vida fundado en el constante mejoramiento económico, social y cultural del pueblo" (DOF, 1993: 5).

\section{Educación y construcción de capacidades en el individuo. Las perspectivas del capital humano y las del desarrollo humano}

Desde el punto de vista teórico, la importancia de mejorar el nivel educativo de la población en general como el fundamento de una mejor calidad de vida, se establece por los enfoques del capital humano y del desarrollo humano, los cuales justifican, en gran medida, los esfuerzos y presupuestos que desde la política pública se llevan a cabo en el ámbito educativo dentro de la política social.

Desde la teoría del capital humano, la educación toma relevancia por las capacidades que brinda a la población de favorecer el desarrollo económico de un país, a través de la creación de una población dotada de las capacidades cognoscitivas y técnicas para contribuir a la producción industrial moderna; mientras que desde el enfoque del desarrollo humano, su importancia radica en contribuir a la eliminación de las privaciones humanas, o a la expansión de las libertades de los individuos para tener una vida plena.

Particularmente, de acuerdo con el enfoque del capital humano desarrollado por teóricos como Schultz (1985), Becker (1983) y Johnson (1972), el desarrollo económico depende esencialmente de la creación de una fuerza de trabajo equipada con la competencia técnica necesaria exigida por la producción industrial moderna, y preparada por su formación para aceptar y promover el progreso económico y técnico. Por lo tanto, los principales postulados de los teóricos del capital humano establecen una relación positiva entre la inversión educativa individual y la percepción futura de ingresos, y entre el gasto gubernamental en educación y el producto nacional, ya que en la metodología de esta corriente de pensamiento el indicador relevante son las llamadas tasas de retorno, que expresan la medida en que el gasto educativo se convierte en valor agregado y por tanto en ganancia.

Por su parte, el enfoque del desarrollo humano, basado esencialmente en las aportaciones intelectuales de Amartya Sen, concibe el desarrollo como un proceso de expansión de las libertades reales de que disfrutan los individuos y exige la eliminación de las principales fuentes de privación de las mismas, como son las carencias sociales, entre las cuales se encuentra la falta de educación o su nivel inadecuado para ejercer estas libertades (Sen, 2000).

Este paradigma centra su atención en las libertades humanas, ya que Sen postula que desde la perspectiva más básica del desarrollo como libertad, la pregunta de si algunas libertades políticas o sociales, como la libertad de participación y disensión políticas o la oportunidad de recibir una educación básica, "contribuyen o no al desarrollo", tiende a pasar por alto una importante cuestión: estas libertades fundamentales (es decir, la libertad de participación política, la oportunidad de recibir una educación o una asistencia sanitaria básicas) se encuentran entre los componentes constitutivos del desarrollo (Sen, 2000).

Desde ambas perspectivas teóricas, los bajos niveles de educación limitan las capacidades del individuo, ya que por un lado no le permiten insertarse de manera adecuada, como mano de obra calificada, en el crecimiento económico de un país, y por otro, le impiden disfrutar de las libertades para tener una vida plena. Por ello, el rezago educativo es visto como un impedimento muy serio para el desarrollo humano sustentable de cualquier nación y por ende debe considerarse como una prioridad para las políticas y acciones gubernamentales y sociales. La educación de adultos, y las instituciones que atienden este rezago, tienen un compromiso social ampliado ante su responsabilidad con la construcción de los sólidos cimientos del desarrollo del país, compromiso que en México aún no se ha percibido ni aceptado en toda su magnitud. 


\section{La educación en el contexto del desarrollo humano sustentable}

El concepto de desarrollo sustentable, definido por la Comisión Mundial del Medio Ambiente y del Desarrollo (CMMAD, 1987: 37), a través del Informe Brundtland, como aquel desarrollo que "satisface las necesidades de las generaciones presentes sin comprometer la capacidad de las generaciones futuras para satisfacer sus propias necesidades", desde su puesta en la palestra pública es un objetivo socialmente generalizado cuya aceptación es casi universal, en parte debido, como lo señala Riechmann (1995), a la diversidad en sus interpretaciones, algunas incompatibles entre sí. ${ }^{2}$

En la interpretación del concepto de desarrollo sustentable se pueden identificar diversas tendencias y múltiples definiciones, desde las esencialmente economicistas, cuya posición más extrema equipara el desarrollo sustentable con el crecimiento económico constante y regular en el tiempo, sin altibajos desequilibradores, y en las que lo ambiental y lo social, o bien no son considerados o se subordinan a lo económico (Riechmann, 1995), hasta las más eclécticas en su enfoque, en las cuales el desarrollo sustentable no se reduce al crecimiento económico, sino que amplía su espectro a la equidad social y al impacto ambiental, proponiendo la articulación de estos tres aspectos.

Dentro de esta corriente heterodoxa de interpretación, la mayoría de las concepciones plantean el desarrollo sustentable como un enfoque basado en pilares: el económico, el social, el ambiental y, en algunas ocasiones, el institucional, destacando las interacciones entre todos éstos (Schuschny y Soto, 2009).

$\mathrm{El}$ consenso internacional en el marco de la ONU, a través del Programa de las Naciones Unidas para el Desarrollo (PNUD), propone el concepto de desarrollo humano sustentable, unificando las nociones previas de desarrollo sustentable derivadas del informe
Bruntland y las aportaciones de Amartya Sen sobre desarrollo humano.

Desde este enfoque, el desarrollo humano sustentable busca expandir la libertad de las personas, mujeres, hombres, niños y niñas, para que puedan elegir lo que quieren ser o hacer, entre distintas oportunidades, y proteger, al mismo tiempo, el medio ambiente.

Para ello, además del crecimiento económico, es necesario distribuir sus beneficios de manera equitativa, regenerar el medio ambiente en lugar de destruirlo, darles voz y capacidad de decisión a las personas en lugar de marginarlas, ampliar sus elecciones y oportunidades, y permitir la participación de las personas en las decisiones que afectan sus vidas. El desarrollo humano sustentable se dirige a la promoción de la dignidad humana y la realización de los derechos humanos, económicos, sociales, culturales, civiles y políticos (PNUD, 2008).

El potencial de la educación en general, como eje articulador de los diversos aspectos del desarrollo humano sustentable, se asienta en el segundo párrafo del artículo tercero constitucional, el cual señala que "La educación que imparta el Estado tenderá a desarrollar armónicamente todas las facultades del ser humano...", y la relevancia en cada uno de los ámbitos del desarrollo humano sustentable se puede establecer de la siguiente manera:

Dimensión económica. El papel de la educación es esencial para propiciar un crecimiento económico que parta de nuevos conocimientos en el campo de las nuevas tecnologías, y para generar soluciones a las viejas problemáticas, incluyendo los problemas sociales y del medio ambiente. Igualmente, el acceso a la educación por parte de mayores sectores de la población, permite ver avances en el desarrollo de un país que van más allá del crecimiento económico y que se traducen en aspectos más cualitativos del mismo.

\footnotetext{
${ }^{2}$ Una discusión completa de la conceptualización de desarrollo sustentable puede verse en E. González-Gaudiano, 2006.
} 
Dimensión social. En la sustentabilidad social, el progreso en materia educativa permite observar en qué medida el avance económico de un país se traduce en desarrollo humano, posibilitando el fortalecimiento de un estilo de desarrollo que procure erradicar la pobreza y la exclusión social, preparando a la población para una mejor inserción en los procesos de desarrollo económico y para un ejercicio más pleno de su ciudadanía.

Dimensión ambiental. En esta arista de la sustentabilidad, la educación es esencial en varios aspectos: en fomentar la conciencia y la participación de la sociedad en el cuidado del medio ambiente, en desarrollar e implementar tecnologías más respetuosas con el entorno, y en el surgimiento y puesta en marcha de regulaciones capaces de ajustar los procesos económicos a las exigencias de los ecosistemas, a fin de que el proceso de desarrollo no rebase la capacidad de carga de éstos.

En general, en la articulación de estos tres aspectos de sustentabilidad, la calidad de la educación en cada uno de sus niveles es fundamental. En particular, la importancia de una educación básica de calidad radica en ser el cimiento de la educación posterior de los individuos, y por lo tanto la base que posibilite el desarrollo de nuevos conocimientos y tecnologías que generen una menor presión sobre el entorno, y la plataforma desde la cual hacer surgir una mentalidad más favorable hacia el cuidado del medio ambiente.

Igualmente, la capacidad de un sistema educativo para brindar una educación básica de calidad a grupos cada vez más amplios de la población contribuye a la justicia y a la equidad social, disminuyendo la pobreza al aumentar las posibilidades de empleo e ingreso para la población, e impactando positivamente en la salud y en la demografía, lo que a su vez puede revertir la tendencia hacia la inequidad en la distribución del ingreso, la reproducción generacional de la pobreza y la presión sobre el medio ambiente.

\section{Delimitación de la educación de adultos en México}

La educación para adultos ${ }^{3}$ comprende, entre otros, los procesos de alfabetización, educación básica, educación para el trabajo, formación para la vida familiar, social y civil, a través de programas educativos orientados a personas jóvenes, a partir de los 15 años, y/o adultas que no tienen desarrolladas las habilidades básicas para leer, escribir y hacer cuentas, o que no iniciaron o concluyeron su educación primaria o secundaria; niños, niñas y jóvenes entre los 10 y 14 años que no están atendidos por el sistema escolarizado de educación primaria; mujeres, indígenas monolingües y bilingües; jóvenes en situación de calle; personas en reclusión; adultos mayores; personas con capacidades diferentes; personas jornaleras agrícolas, migrantes y también la población mexicana que radica en Estados Unidos que no ha iniciado o concluido su educación básica. El Instituto Nacional para la Educación de los Adultos (INEA), tiene la responsabilidad de atender esta modalidad educativa y ofrecer a la población adulta una educación permanente y de buena calidad, que responda a sus aspiraciones y requerimientos, tanto individuales como sociales.

Los servicios de formación para el trabajo enfrentan el reto de dotar a una importante parte de la población mexicana de las capacidades necesarias para participar con las condiciones básicas para su inserción exitosa en el sistema productivo, lo que exige una respuesta integral de las políticas públicas y del aparato educativo a estas necesidades educativas diferenciadas de las del sistema educativo formal, respuestas que hasta ahora han sido insuficientes, como demostraremos en este trabajo, y como lo señaló el ex presidente Felipe Calderón al afirmar que:

\footnotetext{
${ }^{3}$ Instituto Nacional para la Educación de los Adultos (INEA), http://www.inea.gob.mx/index.php/educacionabc.html..
} 


\begin{abstract}
si bien en los últimos años se ha logrado reducir el analfabetismo, esta deficiencia sigue presentándose de manera muy acentuada en varios estados de la república, tanto en el medio rural como entre los pueblos y comunidades indígenas, y el número de personas de 15 años y más que carecen de primaria o secundaria completa es de más de 27 millones. Para dar respuesta a estos desafíos el Instituto Nacional para la Educación de los Adultos centra sus acciones en la atención regionalizada del rezago educativo, a través del Modelo de Educación para la Vida y el Trabajo, la instalación de plazas comunitarias y el desarrollo de estrategias de atención como Cero Rezago y el Programa Oportunidades. ${ }^{4}$
\end{abstract}

\section{Rezago educativo y educación de adultos en México}

El carácter marginal que se le ha dado a la educación para adultos en nuestro país se muestra en la baja incidencia y capacidad para abatir el rezago educativo, dada la escasa cobertura en relación con su población objetivo y la baja proporción de egresos de primaria y secundaria a través de este subsistema. El persistente rezago educativo en México situaba en 2005 a 38.2\% de la población mexicana en situación de desventaja social, ya que por una parte, $29.1 \%$ de la población total se encontraba dentro de este grupo (30 146 664), y el 43.1\% de los niños en edad escolar de entre 3 y 14 años (9 335 009) habitaban en hogares en donde ni el jefe de familia o su cónyuge habían terminado la educación secundaria, situación que, como ya anotamos, impacta en los bajos rendimientos académicos de los alumnos.

La vulnerabilidad social que sufre la población con rezago educativo en nuestro país está relacionada con su insuficiente grado de preparación que le impide insertarse en mejores condiciones a la dinámica socioeconómica actual. En tanto que la vulnerabilidad que genera este rezago en la población en edad escolar, que los sitúa en un entorno familiar con escaso capital socioeducativo, limita su logro educativo.

Lo anterior destaca la necesidad de examinar la situación de la educación para adultos a la luz de la magnitud del rezago educativo de la población mexicana, sus niveles de escolaridad, el número de personas en el INEA realizando su educación básica, los certificados expedidos por el organismo responsable, y la importancia relativa de este gasto en relación con el desembolso educativo federal.

Igualmente, cabe subrayar su relevancia desde el punto de vista del capital humano y del desarrollo humano, como dos enfoques que justifican las inversiones en educación de los gobiernos, dadas las capacidades que los individuos adquieren mediante la educación y de plantear, en general, que la educación para adultos no debe asumirse sólo como una práctica compensatoria de deficiencias del sistema educativo escolarizado, sino como un medio trascendental para expandir el desarrollo educativo de nuestro país y el desarrollo humano sustentable, constituyéndose como un aspecto que contribuye a una calidad educativa integral y sistémica.

\section{La educación no formal y el rezago educativo en México}

La educación no formal o educación para adultos se ha constituido generalmente como una práctica educativa asumida como una actividad compensatoria de deficiencias del sistema educativo formal, y complementaria de programas y políticas encaminadas al desarrollo del medio rural (Pieck-Gochicoa, 1997). Pero, como se ha expuesto, debe asumirse también como un importante medio para potenciar el desarrollo educativo del país y en consecuencia el desarrollo humano sustentable.

Acorde con la perspectiva de que el sector educativo es una dimensión de la actividad humana que requiere la regulación o intervención gubernamental o social y

${ }^{4}$ http://www.informe.gob.mx/informe/pdf/3_3.pdf. 
la adopción de medidas comunes a través de las políticas públicas (Parsons, 2007), en México la educación de adultos se legisló en 1975 y se institucionalizó en 1981, con la creación del Instituto Nacional para la Educación de los Adultos (INEA), el cual atiende a personas mayores de 15 años que no tuvieron la oportunidad de aprender a leer o escribir, o de concluir su primaria o secundaria, enfocándose así a la alfabetización y a la educación básica de adultos. Cabe aclarar que recientemente se ha incluido a la educación media superior como educación básica y por tanto obligatoria, lo que hace que el rezago en este nivel educativo sea ahora también ámbito de trabajo del INEA.

El INEA ha tenido como grupo potencial de atención toda aquella población mexicana de 15 años o más que sea analfabeta, o que no tenga primaria o secundaria terminada, población a la que habría que agregar la que no cuenta con preparatoria o con preparatoria completa, pero que aún no se integra en las cifras, por lo que analizaremos en rezago educativo hasta 2010 donde aún la educación media superior no se consideraba obligatoria en el ámbito del gobierno federal, y lo era sólo en algunas entidades federativas como Jalisco.

De acuerdo con cifras de 2005, esta población ascendía a más de 30 millones de personas y representaba el 43.9\% de la población total de 15 años y más, de las cuales 5.7 millones eran analfabetas, 9.8 millones no contaban con primaria, y 14.6 millones no tenían la secundaria $(19.1 \%, 32.6 \%$ y $48.4 \%$ de la población total en rezago educativo, respectivamente), como se observa en el cuadro 1.

En 2010, esta población aumentó a casi 32 millones de personas y representó el $40.7 \%$ de la población total de 15 años y más, de las cuales 5.4 millones (16.9\% de la población total en rezago educativo) eran analfabetas, es decir se redujo en 300000 personas el analfabetismo, 10.1 millones no contaban con primaria $(31.6 \%)$, lo que significa que la cifra de 2005 aumentó en alrededor de 300000 personas, y 16.4 millones (51.5\%), cifra mayor en casi 2 millones de personas que no tienen la secundaria respecto de 2005; es decir, el 43.8\% de la población de 15 años y más en 2005 se encontraba en rezago educativo y 5 años después el $40.7 \%$ se encontró en esta condición, lo que indica que no se redujo significativamente el rezago educativo, y que los esfuerzos de la política educativa para la educación básica se han centrado en bajar levemente el analfabetismo, pero aumentado la desatención a la población sin primaria ni secundaria completas.

\section{Cuadro 1. Rezago educativo en México}

\begin{tabular}{|l|c|c|c|c|}
\hline \multicolumn{1}{|c|}{ Año } & $\mathbf{2 0 0 5}$ & $\%$ & $\mathbf{2 0 1 0}$ & $\%$ \\
\hline Población de 15 años y más & 68803564 & & 78423336 & \\
\hline Analfabeta & 5747813 & 19.10 & 5393665 & 31.60 \\
\hline Sin primaria & 9825391 & 32.60 & 10082386 & 51.50 \\
\hline Sin secundaria & 14573460 & 48.36 & 16424106 & $\mathbf{3 1 9 0 0 1 5 7}$ \\
\hline Total & $\mathbf{3 0 1 4 6 6 6 4}$ & $\mathbf{4 3 . 8 6}$ & $\mathbf{4 0 . 7 0}$ \\
\hline
\end{tabular}

Fuente: INEA (2010), INEGI (2010). 
Lo anterior, representa un enorme reto no sólo para la institución encargada de la educación especial, el INEA, sino para el gobierno en general, quien tiene la responsabilidad directa y comprometida con la sustentabilidad del desarrollo y del impulso a la competitividad económica y a la lucha por la igualdad social, eje de sus planes y programas de desarrollo.

El rezago educativo se puede observar también si consideramos el nivel máximo de escolaridad de la población entre 25 y 64 años de edad (cuadro 2), ya que constituye una parte de la población económicamente activa (PEA) que requiere el país para producir y crecer, y para tener una ciudadanía responsable y participativa, pero las posibilidades de que esto suceda se limitan, pues en el 2005 el 7.9\% de esta población no contaba con ninguna escolaridad, el 20.0\% sólo tenía educación primaria, el 19.8\% secundaria completa, el 11.2\% educación media superior y el 15.6\% educación superior. Para 2010, el nivel de escolaridad de esta población mejoró ligeramente ya que el 5.8\% no contaba con ninguna escolaridad, el 18.5\% tenía primaria completa, el $22.0 \%$ secundaria completa, el $16.3 \%$ contaba con educación media superior y el 19.2\% con educación superior. Puede observarse que fue leve la reducción del analfabetismo y del porcentaje de población sin escolaridad, y que el esfuerzo educativo se centró en la educación media superior y, moderadamente, en la educación superior.

Sin embargo, la escolaridad de este importante segmento poblacional es insuficiente para los enormes desafíos que enfrenta una economía muy abierta a la competencia exterior, con enormes asimetrías respecto de sus competidores, reflejadas en una de las mayores desigualdades sociales y en un rezago social amplio, y que para superarlos necesita impulsar actividades productivas y sociales de alto valor agregado sustentadas en una población con altos niveles educativos.

También la información del cuadro 2 nos revela que el $41 \%$ de la población en edad de haber concluido estudios superiores, de tener ya una formación universitaria que le posibilite empleo especializado y mejor remunerado, en 2010 no había completado la educación secundaria. Este porcentaje es mayor si consideramos sólo a la población femenina (42.6\%), por lo que la atención al rezago educativo requiere importantes esfuerzos del gobierno y la sociedad para trascender la adversa situación en la que se encuentra más de la mitad de la población de nuestro país.

Lo anterior significa que en 2010, del total de población con edad comprendida entre 25 y 64 años que debería estar ya incorporada a la vida productiva y ciudadana plena, un poco menos de 10 millones no contaban con primaria, 9.4 millones cuentan con primaria completa, y un poco más de millón y medio únicamente tiene secundaria incompleta, lo que suma un poco más de 20 millones de personas que no han completado su educación básica y que son susceptibles de ser atendidos por el subsistema de educación para adultos para concluirla y contar con el mínimo educativo, nueve años de escolaridad.

Esta cantidad es mucho mayor si consideramos, como ya se anotó, que ahora la educación básica incluye a la educación media superior (la preparatoria), donde los más de 11 millones de personas en esta cohorte de edad sólo cuentan con educación secundaria, y no tienen el nivel deseado de 12 años de educación que se ha planteado como aspiración mínima.

El nivel de escolaridad de la PEA de nuestro país tiene un sustento educativo precario y no se ha respondido con políticas públicas enérgicas para elevarlo, pues el subsistema de educación de adultos sigue siendo considerado un complemento menor del sistema de educación básica, cuando tiene la responsabilidad de ofrecer el derecho a la educación a más de 30 millones de personas.

Estas cifras resultan altas si comparamos la necesidad de México de competir como una de las economías más abiertas del mundo, contra otros países como Estados Unidos y Canadá, que no tienen fuerza de trabajo analfabeta y donde la escolaridad promedio de su población, en años de educación formal cursada, es bastante mayor que la de México, por lo 


\begin{tabular}{|c|c|c|c|c|c|c|c|c|c|c|c|c|c|c|c|c|c|}
\hline \multirow{6}{*}{ ণั่ } & \multirow{3}{*}{ 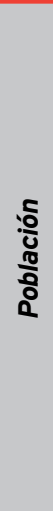 } & $\Sigma$ & $\begin{array}{l}\stackrel{\infty}{\infty} \\
\stackrel{\infty}{\infty} \\
\stackrel{\infty}{\sim} \\
\stackrel{\sim}{\sim}\end{array}$ & $\begin{array}{l}\tilde{\sigma} \\
\alpha \\
\tilde{\rho} \\
\underline{\underline{N}}\end{array}$ & $\begin{array}{l}\text { J } \\
\text { o } \\
\text { N }\end{array}$ & $\begin{array}{l}\text { ఫे } \\
\swarrow \\
o \\
0 \\
0 \\
m\end{array}$ & $\begin{array}{l}\text { Ỹ } \\
\circ \\
\circ \\
\circ \\
\text { ปn }\end{array}$ & $\begin{array}{l}\stackrel{a}{0} \\
\infty \\
m \\
m\end{array}$ & $\begin{array}{l}\infty \\
0 \\
0 \\
\infty \\
\infty \\
0\end{array}$ & 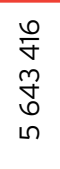 & 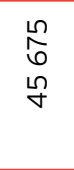 & $\begin{array}{l}\stackrel{\sigma}{\tilde{N}} \\
\infty \\
\stackrel{\sim}{\sim}\end{array}$ & $\frac{\mathbb{z}}{z}$ & 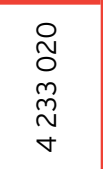 & $\frac{\pi}{z}$ & 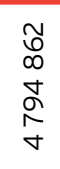 & $\begin{array}{l}\hat{N} \\
\stackrel{N}{\simeq}\end{array}$ \\
\hline & & $I$ & 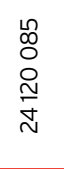 & $\begin{array}{l}\stackrel{\sim}{N} \\
\stackrel{N}{N} \\
\underline{n}\end{array}$ & 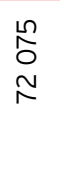 & $\begin{array}{l}\stackrel{N}{0} \\
\stackrel{\sim}{N} \\
\stackrel{N}{m}\end{array}$ & $\begin{array}{l}\stackrel{o}{0} \\
\stackrel{\sim}{\sim} \\
\stackrel{\sim}{\sim} \\
\sim\end{array}$ & $\underset{\substack{N \\
M}}{\stackrel{N}{N}}$ & $\begin{array}{l}\infty \\
\stackrel{0}{0} \\
\sim \\
o \\
\infty \\
\infty\end{array}$ & 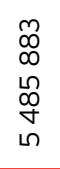 & $\begin{array}{l}\bar{\sigma} \\
\circ \\
\sigma \\
\sigma\end{array}$ & $\frac{\bar{m}}{o}$ & $\frac{\mathbb{z}}{z}$ & $\begin{array}{l}\hat{n} \\
0 \\
\sigma \\
\sigma \\
m\end{array}$ & $\frac{\mathbb{z}}{z}$ & $\begin{array}{l}\overline{1} \\
\stackrel{ }{n} \\
\infty \\
\infty \\
\infty \\
\infty \\
\forall\end{array}$ & $\begin{array}{l}\bar{\sigma} \\
\text { Oे } \\
\stackrel{0}{\simeq}\end{array}$ \\
\hline & & 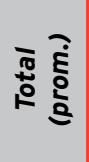 & $\begin{array}{l}0 \\
+ \\
0 \\
o \\
o \\
i n \\
i\end{array}$ & $\begin{array}{l}\mathbb{J} \\
6 \\
\frac{1}{\Omega} \\
\sim\end{array}$ & $\begin{array}{l}\sigma \\
\hat{\sigma} \\
\sigma \\
g \\
\underline{\Xi}\end{array}$ & 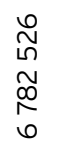 & $\begin{array}{l}\overline{\bar{J}} \\
\bar{\Gamma} \\
\bar{O} \\
\sigma\end{array}$ & $\begin{array}{l}\overline{8} \\
6 \\
6\end{array}$ & \begin{tabular}{l}
0 \\
$ٌ$ \\
$o$ \\
\multirow{H}{n}{} \\
\end{tabular} & $\begin{array}{l}\stackrel{\sigma}{\sigma} \\
\stackrel{N}{N} \\
\stackrel{N}{=} \\
=\end{array}$ & $\begin{array}{l}\stackrel{\infty}{N} \\
\stackrel{+}{\sigma} \\
\sigma\end{array}$ & $\begin{array}{l}\stackrel{p}{\Omega} \\
\alpha \\
m \\
m \\
m\end{array}$ & $\frac{\$}{z}$ & 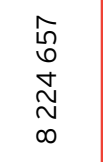 & $\frac{\varangle}{z}$ & $\begin{array}{l}m \\
\bar{f} \\
\delta \\
\infty \\
0 \\
\sigma \\
\sigma\end{array}$ & $\begin{array}{l}\infty \\
\infty \\
\infty \\
\tilde{+} \\
\sim\end{array}$ \\
\hline & & $\Sigma$ & $\underset{\sim}{n}$ & $\stackrel{R}{i}$ & $\stackrel{\stackrel{0}{N}}{\stackrel{0}{0}}$ & $\begin{array}{l}\stackrel{m}{\infty} \\
\stackrel{m}{=}\end{array}$ & $\begin{array}{l}\bar{m} \\
\stackrel{a}{q}\end{array}$ & $\stackrel{\simeq}{0}$ & $\begin{array}{l}\stackrel{\circ}{0} \\
\stackrel{\sim}{~}\end{array}$ & $\stackrel{m}{\stackrel{m}{\sim}}$ & $\stackrel{\circ}{\circ}$ & ò. & $\frac{\mathbb{s}}{z}$ & $\begin{array}{l}8 \\
0 \\
\underline{0}\end{array}$ & $\frac{\mathbb{z}}{\mathrm{z}}$ & $\stackrel{m}{\stackrel{m}{\infty}}$ & \\
\hline & $\therefore$ & $I$ & $\hat{\widetilde{F}}$ & $\begin{array}{l}\infty \\
\infty \\
\stackrel{+}{+}\end{array}$ & $\begin{array}{l}\text { ָे } \\
\text { Oे }\end{array}$ & 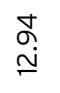 & 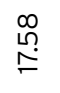 & $\stackrel{m}{\circ}$ & 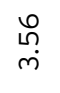 & $\underset{\sim}{\stackrel{N}{N}}$ & શัָ & m. & $\frac{\mathbb{z}}{z}$ & $\begin{array}{l}\stackrel{0}{n} \\
\underline{0}\end{array}$ & $\frac{\mathbb{z}}{z}$ & 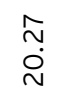 & \\
\hline & & 覀 & & 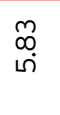 & $\stackrel{\text { ָे }}{\text { Oे }}$ & $\begin{array}{l}\bar{q} \\
\underline{p}\end{array}$ & 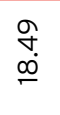 & $\stackrel{m}{0}$ & $\begin{array}{l}\stackrel{\circ}{\infty} \\
\dot{m}\end{array}$ & $\begin{array}{l}\stackrel{\circ}{\text { d }} \\
\stackrel{\sim}{N}\end{array}$ & $\stackrel{\infty}{\circ}$ & $\begin{array}{l}\stackrel{0}{0} \\
\stackrel{0}{0}\end{array}$ & $\frac{\pi}{z}$ & 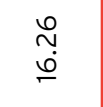 & $\frac{\mathbb{z}}{z}$ & $\frac{n}{\sigma}$ & \\
\hline \multirow{6}{*}{ ஜ̊ } & \multirow{3}{*}{$\begin{array}{l}\delta \\
\frac{0}{3} \\
\frac{\pi}{0} \\
0 \\
0\end{array}$} & $\Sigma$ & $\begin{array}{l}\stackrel{\infty}{\infty} \\
\stackrel{0}{\infty} \\
\stackrel{N}{N} \\
\sim\end{array}$ & 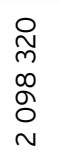 & $\stackrel{\mathbb{z}}{\mathbf{z}}$ & 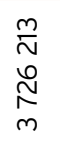 & $\begin{array}{l}\tilde{\gamma} \\
\stackrel{+}{\sigma} \\
\infty \\
\infty \\
\infty \\
\sigma\end{array}$ & $\frac{\mathbb{z}}{z}$ & $\begin{array}{l}\bar{D} \\
\stackrel{N}{N} \\
\tilde{\sigma}\end{array}$ & $\begin{array}{l}\stackrel{0}{\sim} \\
\stackrel{\infty}{\infty} \\
\stackrel{\sim}{+}\end{array}$ & $\stackrel{\$}{z}$ & 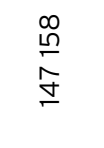 & $\begin{array}{l}\tilde{N} \\
\tilde{n} \\
\stackrel{m}{m} \\
\stackrel{n}{n}\end{array}$ & 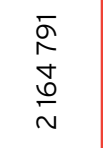 & $\begin{array}{l}\text { ్ָ } \\
\text { J } \\
\text { ָे }\end{array}$ & $\begin{array}{l}\frac{\bar{y}}{\infty} \\
\stackrel{\infty}{\oplus} \\
m\end{array}$ & 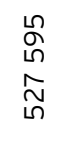 \\
\hline & & $I$ & $\begin{array}{l}\hat{N} \\
\sim \\
\infty \\
\infty \\
0 \\
\sim\end{array}$ & 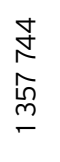 & $\frac{1}{z}$ & $\begin{array}{l}\bigcirc \\
\stackrel{2}{0} \\
\infty \\
\infty \\
o \\
m\end{array}$ & 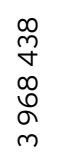 & $\frac{\mathbb{z}}{z}$ & 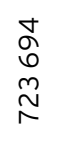 & $\begin{array}{l}\stackrel{0}{\sim} \\
\stackrel{+}{+} \\
\stackrel{\sim}{\sim} \\
\stackrel{+}{+}\end{array}$ & $\stackrel{\$}{z}$ & $\begin{array}{l}\underset{\sim}{N} \\
\underset{\sim}{N}\end{array}$ & \begin{tabular}{l}
$\stackrel{N}{N}$ \\
$\infty$ \\
$\sigma$ \\
\multirow{\forall}{\sigma}{}
\end{tabular} & 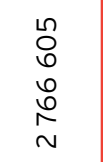 & $\begin{array}{l}\stackrel{\llcorner}{\Omega} \\
\stackrel{\sim}{\Omega} \\
\llcorner\end{array}$ & $\begin{array}{l}\frac{1}{0} \\
\sim \\
\sim \\
0 \\
0 \\
m\end{array}$ & 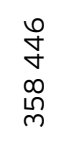 \\
\hline & & 胥 & 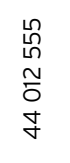 & 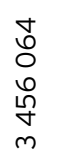 & $\frac{\mathbf{r}}{\mathrm{z}}$ & $\begin{array}{l}\frac{n}{0} \\
\stackrel{n}{n} \\
\infty \\
\infty \\
0\end{array}$ & $\begin{array}{l}0 \\
\tilde{\sigma} \\
\varnothing \\
\varnothing \\
\infty \\
\infty\end{array}$ & $\underset{z}{z}$ & $\begin{array}{l}\stackrel{n}{n} \\
\infty \\
\infty \\
\stackrel{n}{m} \\
\stackrel{-}{-}\end{array}$ & $\begin{array}{l}N \\
\infty \\
0 \\
ٌ م \\
م \\
\infty \\
\infty\end{array}$ & $\frac{\varangle}{z}$ & $\begin{array}{l}\stackrel{0}{\infty} \\
\stackrel{+}{+} \\
\stackrel{2}{上}\end{array}$ & 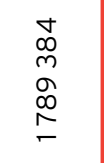 & $\begin{array}{l}\stackrel{0}{ } \\
\stackrel{m}{0} \\
\frac{0}{\alpha} \\
\sigma\end{array}$ & $\begin{array}{l}\underset{\widetilde{J}}{\infty} \\
\infty \\
\infty \\
\infty\end{array}$ & $\begin{array}{l}0 \\
\stackrel{2}{\Lambda} \\
0 \\
0 \\
\infty \\
0\end{array}$ & $\begin{array}{l}\bar{J} \\
0 \\
0 \\
\infty \\
\infty\end{array}$ \\
\hline & \multirow{3}{*}{ ¿ } & $\Sigma$ & 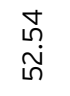 & $\begin{array}{l}\text { ò } \\
\text { o. }\end{array}$ & $\stackrel{\mathbb{L}}{z}$ & $\underset{\underline{0}}{\overline{0}}$ & $\begin{array}{l}\tilde{N} \\
\text { ळे }\end{array}$ & $\stackrel{\mathbb{z}}{z}$ & $\stackrel{\circ}{\sim}$ & $\begin{array}{l}\stackrel{+}{\sigma} \\
\infty \\
\infty\end{array}$ & $\stackrel{\varangle}{z}$ & 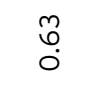 & $\begin{array}{l}\stackrel{\sigma}{\hat{n}} \\
\stackrel{n}{ }\end{array}$ & $\stackrel{\stackrel{n}{m}}{\sigma}$ & $\begin{array}{l}\infty \\
\stackrel{n}{0} \\
0\end{array}$ & $\underset{\stackrel{N}{N}}{\stackrel{N}{m}}$ & $\stackrel{\infty}{\sim}$ \\
\hline & & $I$ & 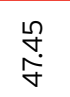 & $\stackrel{\text { பூ }}{\emptyset}$ & $\frac{\mathbb{z}}{\mathrm{z}}$ & 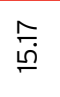 & $\begin{array}{l}\circ \\
\stackrel{0}{\circ}\end{array}$ & $\frac{\mathbb{a}}{z}$ & $\stackrel{0}{\stackrel{0}{*}}$ & $\begin{array}{l}\stackrel{p}{2} \\
\stackrel{i}{N}\end{array}$ & $\stackrel{\$}{z}$ & $\stackrel{m}{\circ}$ & $\stackrel{n}{\sim}$ & $\stackrel{\stackrel{\sim}{\sim}}{\stackrel{m}{\sim}}$ & $\stackrel{\stackrel{0}{0}}{0}$ & $\stackrel{\Re}{\stackrel{n}{\ominus}}$ & 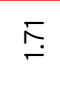 \\
\hline & & $\begin{array}{l}\overline{\mathfrak{\varepsilon}} \\
\stackrel{5}{\circ} \\
\stackrel{0}{0}\end{array}$ & & 용 & $\frac{\mathbb{z}}{\mathrm{z}}$ & $\begin{array}{l}\stackrel{\circ}{\circ} \\
\stackrel{\leftrightarrow}{\llcorner}\end{array}$ & $\begin{array}{l}\overline{0} \\
\stackrel{\sim}{\sim}\end{array}$ & $\underset{z}{z}$ & $\stackrel{\infty}{\stackrel{\infty}{\sim}}$ & $\begin{array}{l}\hat{N} \\
\sigma\end{array}$ & $\stackrel{\varangle}{z}$ & 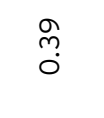 & $\begin{array}{l}\stackrel{0}{\circ} \\
\stackrel{+}{*}\end{array}$ & $\stackrel{\stackrel{m}{\rightleftharpoons}}{=}$ & $\stackrel{m}{\text { m }}$ & $\begin{array}{l}\infty \\
\stackrel{\infty}{n} \\
\stackrel{n}{n}\end{array}$ & $\underset{\sim}{\sim}$ \\
\hline & & & 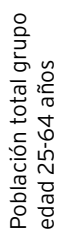 & 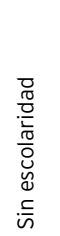 & 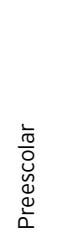 & 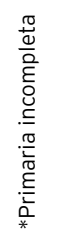 & 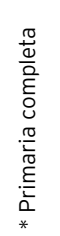 & 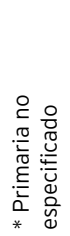 & 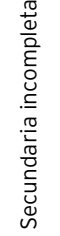 & 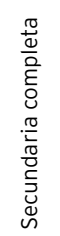 & 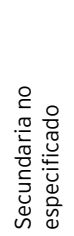 & 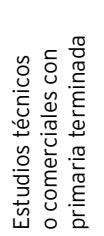 & 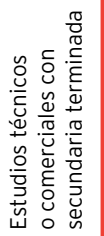 & 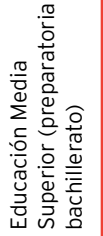 & 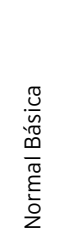 & 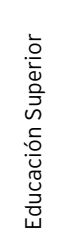 & 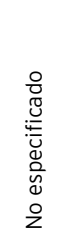 \\
\hline
\end{tabular}


cual el sistema educativo debe cumplir con su compromiso social de asegurar el derecho a la educación y proporcionar las condiciones de competitividad económica e igualdad social que requieren las actuales y futuras condiciones productivas y sociales.

\section{Evolución de la educación básica para adultos}

No obstante la gran cantidad de adultos sin educación básica, y que la importancia de la educación para el desarrollo humano sustentable del país se reconoce en los planes y programas de gobierno, las cifras de alumnos inscritos en primaria y secundaria en el INEA y en los centros de Educación Básica (CEBAS) muestran que no se considera estratégico el subsistema de educación de adultos, pues revelan que en 2005 el número de personas atendidas en primaria y secundaria (304 377 y 810 147, respectivamente), representan apenas el $2.9 \%$ y el $4.0 \%$ de la población de entre 25 y 64 años susceptible de recibirlas; en 2010, los 330241 atendidos en primaria eran el 3.3\% y los 788538 de secundaria representaban apenas el 3.8 por ciento. Este nivel de baja atención y acceso a la educación de adultos es un factor más de exclusión e inequidad social, y un lastre para el avance de México hacia la conformación de una fuerza laboral altamente calificada que pueda acceder a empleo digno $^{5}$ y con ello remontar la pobreza.

Los resultados son más desalentadores, si comparamos las cifras de personas entre 25 y 64 años sin educación primaria y sin secundaria, con el número de certificados de educación básica expedidos por el INEA ese año (cuadro 4), donde el total de personas que obtuvieron su certificado de primaria representaron el $1.6 \%$ de los que no contaban con ese nivel educativo, en tanto que las que obtuvieron su certificado de secundaria representaban el 2.9\% de las personas con sólo primaria. Esto nos lleva a la conclusión de que la efectividad del sistema es muy baja para satisfacer las enormes y crecientes necesidades de este tipo de educación, cuya atención no puede seguir posponiéndose, a riesgo de no avanzar en la ruta del desarrollo humano sustentable.

Adicionalmente, se observa que la población atendida por este subsistema (cuadro 3) en educación primaria durante el periodo 2000-2010, muestra una tendencia a la baja (29.9\%), con excepción de 2007, año en el que el número de personas con educación primaria superó el nivel de 2000 en un 49.8\%. Igualmente, si comparamos las cifras de certificados de primaria expedidos, se observa una caída de $17.9 \%$, de 2000 a 2010. Estas cifras reflejan no sólo la reducida atención que se da a quienes tienen derecho a la educación, sino además, la baja eficiencia terminal del subsistema que ni siquiera gradúa a la mitad de quienes se inscriben en primaria. La tasa de crecimiento promedio de atención en educación secundaria por este subsistema, creció en el periodo considerado en un $24.2 \%$ y la expedición de certificados para este nivel lo hizo sólo en un 8.0\%, observándose también que en este nivel se comparte la baja eficiencia terminal del nivel educativo previo.

La limitada atención que el subsistema de educación de adultos da a la población entre 25 y 64 años de edad, aunado a la alta proporción de personas inscritas en él que no logran finalizar sus estudios, muestra la urgente necesidad de transformar este subsistema educativo y replantear su condición de complementario del sistema educativa a estratégico para el desarrollo nacional.

\section{Evolución del gasto público en educación e importancia relativa del gasto en otros}

Otra muestra más de que el Estado ha desestimado la educación para adultos es la evolución del gasto

\footnotetext{
${ }^{5}$ La propuesta de la Organización Internacional del Trabajo (OIT) denomina trabajo decente a aquél "trabajo digno y productivo en condiciones de libertad, equidad, seguridad y dignidad, en el cual los derechos son protegidos y que cuenta con remuneración adecuada y protección social” (OIT, 1999: 4), condición que no se logra cuando se tiene un nivel educativo precario.
} 
Cuadro 3. Educación básica para adultos. Personas, 2000-2010

\begin{tabular}{|c|c|c|}
\hline Año & Primaria & Secundaria \\
\hline 2000 & 470931 & 635028 \\
\hline 2001 & 376947 & 620037 \\
\hline 2002 & 394393 & 681183 \\
\hline 2003 & 323923 & 718512 \\
\hline 2004 & 276209 & 750393 \\
\hline 2005 & 304377 & 810147 \\
\hline 2006 & 296055 & 814096 \\
\hline 2007 & 705474 & 1573604 \\
\hline 2008 & 220188 & 446633 \\
\hline 2009 & 323287 & 802673 \\
\hline 2010 & 323287 & 788538 \\
\hline
\end{tabular}

Cuadro 4. Educación básica para adultos. Certificados expedidos, 2000-2010

\begin{tabular}{|c|c|c|}
\hline Año & Primaria & Secundaria \\
\hline 2000 & 188751 & 305458 \\
\hline 2001 & 166064 & 286127 \\
\hline 2002 & 144286 & 252160 \\
\hline 2003 & 155431 & 285606 \\
\hline 2004 & 160299 & 332862 \\
\hline 2005 & 161141 & 326814 \\
\hline 2006 & 203201 & 423463 \\
\hline 2007 & 155421 & 331782 \\
\hline 2008 & 177207 & 365463 \\
\hline 2009 & 169935 & 346260 \\
\hline 2010 & 155021 & 329780 \\
\hline
\end{tabular}

Fuente: SEP (2011) 
educativo en el rubro de otros, en el cual se incluye el gasto destinado a la capacitación para el trabajo, alfabetización, educación primaria y secundaria para adultos, al fomento de la cultura y el deporte, así como los gastos de la administración central. Este rubro disminuyó, en términos reales, en 39.1\% de 1994 a 2010; asimismo, decreció en $14.1 \%$ como porcentaje del gasto federal en educación al pasar de representar el $21.5 \%$ a sólo el $7.4 \%$ del gasto educativo federal total. Lo anterior, pese a que el gasto educativo federal total se incrementó en $76.2 \%$ en términos reales en el mismo periodo (véase cuadro 5), siendo el único rubro cuyo monto cayó en términos reales, ya que durante el mismo periodo el gasto en educación básica, media y superior aumentaron en $84.9 \%, 68.5 \%$ y $81.9 \%$ respectivamente, lo que demuestra el abandono relativo que presupuestalmente está teniendo este subsistema.

De lo anterior se desprende que el gasto destinado a la educación de los adultos significa proporcionalmente un monto muy por debajo del gasto público de cualquier otra modalidad educativa, ya que no representa una proporción importante dentro del total del gasto educativo federal a pesar de que la población con rezago educativo es una proporción importante de la población de nuestro país. Al respecto, el Observatorio Ciudadano de la Educación (OCE, 2000), señala que en México la política educativa ha soslayado la educación para adultos, al situarla en una posición marginal, ya que ésta es impartida por personal voluntario sumamente móvil e insuficientemente calificado en instalaciones inadecuadas (generalmente prestadas); de ahí su escasa cobertura y eficiencia; opinión con la que coinciden otros autores como PieckGochicoa (1997) y Hernández-Flores (2008).

No obstante que los recursos públicos destinados a la educación para adultos son una limitación significativa para ésta, los recortes presupuestales siguen afectando esta actividad de manera importante; ya que el presupuesto del INEA para 2010 se redujo en casi 300 millones de pesos con respecto a 2009, al pasar de 2095 millones a 1871 millones. $^{6}$

Al respecto, el informe del relator especial sobre el Derecho a la Educación de la Comisión de Derechos Humanos de la $\mathrm{ONU}^{7}$ Vernon Muñoz Villalobos, en su visita oficial a México en febrero de 2010, señaló que es importante que se fortalezca la institución encargada de la educación para las personas adultas, pues no obstante el número de población que tiene que atender el subsistema, apenas recibe un $0.86 \%$ del presupuesto de la Secretaría de Educación Pública (SEP $)^{8}$ y está en manos de "asesores solidarios" (100 mil personas) que trabajan con mucho entusiasmo a tiempo parcial sin contar con formación pedagógica superior.

\section{Relación entre el nivel educativo de padres con el logro académico de los hijos}

En estudios empíricos realizados durante las últimas cuatro décadas sobre los determinantes del rendimiento académico, se ha encontrado una relación directa entre el nivel educativo de los padres y el logro educativo de los hijos (véase por ejemplo Brunner y Elaqua, 2003), lo cual implica que la educación de los adultos en general, no sólo es una medida que compensa el rezago de la población objetivo, sino que imprime un impulso positivo en la educación de los hijos, especialmente el nivel educativo de la madre (PISA 2003 y 2006 en OCDE, 2004, 2007a y 2007b), por lo que tiene especial relevancia para las bases del desarrollo humano sustentable.

De acuerdo con estos estudios, se identifica que el impacto de la educación de los padres en la educación de los hijos se da en dos sentidos; por un lado se

\footnotetext{
${ }^{6}$ http://www.sep.gob.mx/wb/sep1/resumen080310 [consulta: 8 de abril de 2010].

${ }^{7}$ Muñoz, Vernor (2010).

${ }^{8}$ Porcentaje muy por debajo del 6\% recomendado en la Declaración de Bonn sobre la Financiación de la Educación de Adultos para el Desarrollo (DVV International et al., 2009).
} 
Cuadro 5. Gasto educativo federal total y en otros, 1994-2010 (millones de pesos a precios de 2003)

\begin{tabular}{|c|c|c|c|}
\hline Año & Total (1) & Otros (2)* & $\begin{array}{c}\text { Partic. \% } \\
\text { (2) / (1) X } 100\end{array}$ \\
\hline 1994 & 210796 & 45258 & 21.5 \\
\hline 1995 & 218043 & 37299 & 17.1 \\
\hline 1996 & 235055 & 37026 & 15.8 \\
\hline 1997 & 246001 & 37990 & 15.4 \\
\hline 1998 & 267066 & 18195 & 6.8 \\
\hline 1999 & 272473 & 21777 & 8.0 \\
\hline 2000 & 286796 & 21637 & 7.5 \\
\hline 2001 & 289345 & 19150 & 6.6 \\
\hline 2002 & 294658 & 20773 & 7.1 \\
\hline 2003 & 307407 & 21849 & 7.1 \\
\hline 2004 & 308799 & 21636 & 7.0 \\
\hline 2005 & 324588 & 22944 & 7.1 \\
\hline 2006 & 343837 & 29184 & 8.5 \\
\hline 2007 & 350751 & 27408 & 7.8 \\
\hline 2008 & 366102 & 23908 & 6.5 \\
\hline 2009 & 371746 & 34503 & 9.3 \\
\hline 2010 & 371365 & 27550 & 7.4 \\
\hline
\end{tabular}

* Rubro donde se ubica el presupuesto para la Educación de Adultos. Fuente: INEE (2011). 
presupone que es posible incrementar el rendimiento académico de los hijos aumentando el nivel de escolaridad de los padres, es decir, se establece una relación positiva entre el nivel educativo de los padres y el logro académico de los hijos: a mayor nivel de escolaridad de los padres mayor rendimiento escolar de los hijos y viceversa; por otro lado, la educación de los padres incentiva la educación de la generación siguiente, demostrando que los hijos de padres que han superado la enseñanza básica, tienen más posibilidades de terminar ese ciclo educativo.

De lo anterior se desprende que, en general, los rendimientos académicos más deficientes y la mayor deserción escolar en los diversos niveles de escolaridad se asocian con niñas y niños provenientes de hogares encabezados por padres con baja escolaridad. Pese a lo mencionado arriba, y debido al enorme rezago educativo, en 2010 el 73.2\% de los niños entre 3 y 14 años habitaban en hogares donde el jefe de familia o cónyuge no alcanzaban la educación media superior, ahora también considerada obligatoria (cuadro 6).

El cuadro anterior evidencia la necesidad de que el Estado mexicano diseñe mejor las políticas públicas y busque mecanismos a través de los cuales los adultos, que son o serán padres, alcancen al menos la educación básica para estar en posibilidad de contribuir más significativamente en la educación de sus hijos, y para que esta responsabilidad no recaiga únicamente en las escuelas y sus docentes. Dichos mecanismos estarían en concordancia con los objetivos de universalizar la educación básica y mejorar la calidad de la educación en la niñez, ya que mejorarían en sí mismos los niveles de escolaridad de la población e incidirían en el logro educativo de la siguiente generación, fortaleciéndose así las bases de la sustentabilidad de nuestro desarrollo como nación.

En este sentido se pronuncia el INEE (2007), al señalar que, de acuerdo con lo establecido por la Ley General de Educación en su artículo cuarto, que indica que todos los habitantes del país deben cursar la educación preescolar, primaria y secundaria (y a partir de 2012 la educación media superior), es posible establecer como meta que todas las niñas y niños habiten en hogares donde al menos uno de los padres tenga la educación básica completa, ya que quienes habitan en hogares donde el máximo nivel de escolaridad de los responsables del hogar es menor a secundaria, se encuentran por debajo del nivel mínimo deseable, colocándolos en una situación de mayor vulnerabilidad educativa y con ello limitando

\section{Cuadro 6. Hijos de 3 a 14 años, según máximo nivel educativo alcanzado por el jefe del hogar o su cónyuge, a nivel nacional}

\begin{tabular}{|l|c|c|}
\hline \multicolumn{1}{|c|}{ Nivel educativo } & \multicolumn{2}{c|}{2010} \\
\hline Sin escolaridad & $\%$ & 2069996 \\
\hline Educación básica & 7.86 & 17197069 \\
\hline Educación media superior & 65.31 & 3657801 \\
\hline Educación superior & 13.89 & 3324834 \\
\hline Total de hijos de 3 a 14 años & 12.62 & $\mathbf{2 6 3 3 0 1 9 3}$ \\
\hline
\end{tabular}

Fuente: INEGI (2010). 
las posibilidades de su desarrollo humano y de su contribución al desarrollo nacional.

\section{Conclusiones}

Ampliar el acceso de la población con rezago educativo al subsistema de educación para adultos es una meta insatisfecha por parte de la política educativa del país, que conlleva aspectos como el de la baja cobertura, gran deserción y poca eficiencia y requiere su urgente atención.

La persistencia de una alta proporción de población adulta sin educación básica en nuestro país es una limitante para el desarrollo humano sustentable, ya que dificulta la articulación de los diversos órdenes del mismo; esto es, el desarrollo económico, la equidad social y el respeto al medio ambiente, los cuales requieren aumentar cada vez más las capacidades humanas, sociales y productivas sobre competencias elementales y capacidades básicas que debería proporcionar la educación básica para adultos.

Estas limitaciones se observan en varios aspectos de la vida de los individuos; en primer lugar, las decisiones que tienen que ver con su bienestar personal y con el de su familia, como las relacionadas con la procreación, estilo de vida sano, y educación de los hijos, que ante el bajo nivel educativo están siendo tomadas sin mucho conocimiento de causa; en segundo lugar, el desarrollo de principios y valores cívicos es insuficiente para ejercer de una manera plena su ciudadanía y participar en asuntos públicos que les afectan directamente.

En el ámbito más general, una proporción considerable de adultos sin educación básica contribuye escasamente y de forma frágil al crecimiento económico, lo que dificulta las posibilidades de un mayor desarrollo social, ya que restringe el crecimiento de los niveles de empleo, de los salarios y de los recursos destinados a los programas de atención social, incluyendo la educación; creándose un círculo vicioso en el que, además, se refuerza la inadecuada formación de actitudes y valores cívicos; lo anterior limita la solidaridad y cohesión social, el respeto al estado de derecho y la participación democrática.

Por ello, es necesario formular políticas públicas en el ámbito educativo que fortalezcan la educación para adultos y que propicien que el concepto de la calidad de la educación básica centrada en la educación formal y en la escuela, como concepto socialmente construido, vaya más allá de cualquier prueba estandarizada (EXCALE, ENLACE, PISA, etcétera), y avance hacia una definición más amplia y multifacética, con una visión sistémica de la misma, en la que los beneficios de la educación se hagan extensibles a todos los actores sociales, lo que significaría un golpe de timón a las actuales concepciones de la evaluación de la calidad educativa y de las políticas públicas para mejorarla.

En el ámbito de la educación para los adultos, estas políticas públicas deben responder más a las necesidades y expectativas de los educandos, actualizando los contenidos, mejorando la capacitación de los agentes educativos, actualizando los modelos pedagógicos para adecuarlos a las circunstancias particulares de la población objetivo, y vinculando más dicha educación con el mercado de trabajo y la vida productiva.

Es necesario diversificar y ampliar considerablemente la oferta de educación para adultos que incluya acciones afirmativas como extender programas que apoyen a mujeres embarazadas o madres jóvenes, para que concluyan su educación básica y continúen una formación para el trabajo, incluso con mayores compensaciones económicas, dado el rol fundamental que aún tienen las mujeres en la formación de sus hijos y en la influencia sobre la educación de la familia.

El desarrollo humano sustentable no podrá alcanzarse sin la puesta en marcha de una decidida política educativa encaminada a lograr la máxima cobertura de la educación básica en los adultos, que permita mejorar la calidad de la educación básica de sus hijos y posibilite un mayor acceso a la educación media y superior a grupos más amplios de la población y constituya un fundamento sólido para el desarrollo humano sustentable de México. 


\section{Referencias}

Becker, Gary S. (1983), "Inversión en capital humano e ingresos", en El mercado de trabajo: teoría y aplicaciones, Madrid, Alianza Editorial, pp. 39-63.

Brunner, Joaquín y Gregory Elacqua (2003), Factores que inciden en una educación efectiva. Evidencia internacional, Santiago de Chile, GEPAL (Serie de Políticas Sociales, 9), http:// www.educoas.org/portal/bdigital/lae-ducacion/139/ pdfs/139pdf1.pdf [consulta: septiembre 2008].

Comisión Mundial del Medio Ambiente y el Desarrollo (CMMAD) (1989), Our common future, from one earth to one world. Nuestro futuro común, de una tierra para un mundo. Informe Brundtland, Nueva York, ONU, http://www. un-documents.net/ocf-ov.htm\#I.3 [consulta: febrero 2008].

Diario Oficial de la Federación (1993), Artículo tercero constitucional, http://info4.juridicas.unam.mx/ijure/ tcfed/9.htm?s [consulta: 2 de diciembre 2013].

Díaz-Gutiérrez, María Antonieta, Gustavo FloresVázquez y Felipe Martínez-Rizo (2007), PISA 2006 en México, México, Instituto Nacional para la Evaluación de la Educación.

DVV International, ICAE, EAEA, ASPBAE, BoCAED, One World, UNESCO/UIL (2009), Declaración de Bonn sobre financiación de la educación de personas adultas para el desarrollo, Bonn, Instituto de Cooperación Internacional de la Asociación Alemana para la Educación de Adultos/DVV International, http://www.iiz-dvv.de/ index.php?article_id $=1003 \&$ clang $=3$ [consulta: agosto 2013].

González-Gaudiano, Édgar (2006), "Las ciencias sociales y la sustentabilidad", en Desarrollo sustentable: diagnóstico y prospectiva para Nuevo León, Colección Desarrollo Sustentable, vol. 1, México, Instituto de Investigaciones Sociales-UANL/Plaza y Valdés, pp. 17-37.

Hernández-Flores, Gloria (2008), "Un año de encuentros en educación de personas jóvenes y adultas en México", en Revista Interamericana de Educación de Adultos, año 30, núm. 1, México, Centro de Cooperación Regional para la Educación de Adultos en América Latina y el Caribe (CREFAL), http://tariacuri.crefal.edu.mx/ rieda/ene_jun_2008/pdf/transiciones.pdf [consulta: agosto 2013].

Honorable Congreso de la Unión (1917), Constitución política de los Estados Unidos Mexicanos, Querétaro, DOF 5-02-1917, http://www.diputados.gob.mx/ LeyesBiblio/pdf/1.pdf [consulta: agosto 2013].

Honorable Congreso de la Unión (2006), Ley General de Educación, México, DOF 22-06-2006, http://www.oei. es/quipu/mexico/Ley_gen_educ.pdf [consulta: julio 2007].

Instituto Nacional para la Educación de los Adultos (INEA) (2010), Rezago de la Población de 15 años y más en educación básica, México, INEA, http://www.inea.gob. $\mathrm{mx} /$ transparencia/pdf/rezago_censo2010_nd.pdf [consulta: agosto 2013].

Instituto Nacional para la Evaluación de la Educación (INEE) (2010), Panorama educativo de México. Indicadores del Sistema Educativo Nacional 2010, Anexo electrónico AR03a, México, INEE, http://www.inee.edu.mx/bie_ wr/mapa_indica/2010/PanoramaEducativoDeMexico/ AR/AR03/2010_AR03_a-vinculo.pdf [consulta: marzo $2010]$.

INEE (2007), Panorama Educativo de México. Indicadores del Sistema Educativo Nacional 2007, México, INEE, http:// www.inee.edu.mx/index.php/70-publicaciones/ panorama-educativo-capitulos/423-panoramaeducativo-de-mexico-indicadores-del-sistemaeducativo-nacional-2007 [consulta: marzo 2010].

INEE (2006a), Panorama Educativo de México. Indicadores del Sistema Educativo Nacional 2006, México, INEE, http://www.inee.edu.mx/index.php/transparencia/ bienes-de-activo-fijo-del-inee/doc_details/43metodologia-para-la-administracion-de-riesgos-delinee?tmpl=component [consulta: marzo 2010].

INEE (2006b), Panorama Educativo de México. Indicadores del Sistema Educativo Nacional 2006 Resultados de españoly las matemáticas en la educación básica en México. Sexto de Primaria y Tercero de Secundaria, México, INEE, http://www.inee. 
edu.mx/images/stories/Publicaciones/Panorama_ educativo/2006/Partes/panorama200609.pdf [consulta: marzo 2010].

Instituto Nacional de Estadística Geografia e Informática (INEGI) (2005), II Conteo de población yvivienda, México, INEGI, http://www3.inegi.org.mx/sistemas/TabuladosBasicos/ tabentidad.aspx?c=33145\&s=est [consulta: marzo 2010].

Muñoz, Vernor (2010), "Informe de Vernor Muñoz sobre el derecho a la educación en México", en La Carta CEAAL, núm. 359, Panamá, Consejo de Educación de Adultos de América Latina, http://www.gloobal.net/iepala/ gloobal/fichas/ficha.php? entidad=Textos\&id $=10376$ [consulta: abril 2010].

Observatorio Ciudadano de la Educación (OCE) (2000), "La educación de adultos en búsqueda de un nuevo modelo", en Comunicados OCE, núm. 29, México, OCE/IISUE-UNAM, http://www.observatorio.org/ comunicados/comun029_2.html [consulta: febrero 2010].

Organización para la Cooperación y el Desarrollo Económicos (OCDE) (2007a), PISA 2006. Science competencies for tomorrow's world. Volume-1 Analysis, París, OCDE, http://www.oecd.org/ dataoecd/30/17/39703267.pdf [consulta: diciembre 2007].

OCDE (2007b), PISA 2006. Science competencies for tomorrow's world. Volume-2: data, París, OCDE, http://www.oecd.org/ dataoecd/30/18/39703566.pdf [consulta: diciembre 2007].

OCDE (2004), Primeros resultados de PISA 2003. Resumen Ejecutivo, París, OCDE, http://www.oei.es/quipu/ mexico/informe_pisa2003.pdf [consulta: julio 2007].

Organización Internacional del Trabajo (OIT) (1999), Trabajo decente, Ginebra, Memoria del Director General a la $87^{\mathrm{a}}$ reunión de la Conferencia Internacional del Trabajo.

Organización de las Naciones Unidas (ONU) (2000), Objetivos de desarrollo del milenio, Nueva York, Departamento de Información Pública de las Naciones Unidas, http://www.un.org/es/millenniumgoals/ bkgd.shtml [consulta: abril 2010].
Parsons, Wayne (2007), Políticas públicas. Una introducción a la teoría y la práctica del análisis de las políticas públicas, Argentina, FLACSO-México.

Pieck-Gochicoa, Enrique (1997), La educación para los adultos: caracterización, impacto y consideraciones propositivas, Colección Documentos de Investigación, núm. 3, Zinacantepec, El Colegio Mexiquense A. C., http:// www.cmq.edu.mx/index.php/component/docman/ doc_download/143-di0030096.html [consulta: febrero 2010].

Programa de las Naciones Unidas para el Desarrollo (PNUD) (2008), Informe de actividades del Programa de las Naciones Unidas para el Desarrollo en México. Desarrollo humano para vivir mejor, México, PNUD, http://www. undp.org.mx/IMG/pdf/Informe_PNUDMx.pdf [consulta: agosto 2013].

Presidencia de la República (2007), Plan Nacional de Desarrollo 2007-2012, México, DOF 31-05-2007, http://dof.gob.mx/nota_detalle.php?codigo $=498940$ $1 \&$ fecha=31/05/2007 [consulta: enero 2010].

Reynoso-Cantú, E. L., E. Marúm-Espinosa (2010), "La educación secundaria como uno de los sustentos del desarrollo humano sustentable de México", en Revista Educación y Ciencia [cuarta época], vol. 1, núm. 37, Mérida, Facultad de Educación de la Universidad Autónoma de Yucatán, pp. 73-90.

Riechmann, J. (1995), "Desarrollo sostenible: la lucha por la interpretación", en De la economía a la ecología, Madrid, Trotta, pp. 11-35.

Schultz, Theodore W. (1985), Invirtiendo en la gente, Barcelona, Ariel, pp.13-44.

Schuschny, A. y H. Soto (2009), Guía metodológica. Diseño de indicadores compuestos de desarrollo sostenible, Santiago de Chile, CEPAL, ONU.

Sen, A. (2000), Desarrollo y libertad, Barcelona, Planeta. Secretaría de Educación Pública (SEP) (2011), Sistema Educativo de los Estados Unidos Mexicanos, principales cifras ciclo escolar 2010-201 1, México, SEP, http://www. sep.gob.mx/work/models/sep1/Resource/1899/2/ images/principales_cifras_2010_2011.pdf [consulta: marzo 2010]. 
SEP (2008a), Sistema educativo de los Estados Unidos Mexicanos, principales cifras, ciclo escolar 2006-2007, México, SEP, http://www.dgpp.sep.gob.mx/Estadi/Principales $\% 20$ cifras\%202006-2007\%20grisl.pdf [consulta: febrero 2012].

SEP (2008b), Sistema Educativo de los Estados Unidos Mexicanos, principales cifras, ciclo escolar 2007-2008,
México, SEP, http://www.dgpp.sep.gob.mx/Estadi/ Principales_cifras_2007_2008.pdf [consulta: marzo 2010].

SEP (2007), Programa Sectorial de Educación, México, SEP, http://upepe.sep.gob.mx/prog_sec.pdf [consulta: enero 2008].

\section{Cómo citar este artículo:}

Marúm-Espinosa, Elia y Elsa-Laura Reynoso-Cantú (2014), “La importancia de la educación no formal para el desarrollo humano sustentable en México", en Revista Iberoamericana de Educación Superior (RIES), México, UNAM-IISUE/Universia, vol. V, núm. 12, pp. 137-155, http://ries.universia.net/ index.php/ries/article/view/444 [consulta: fecha de última consulta]. 